\title{
Cinética de las Características Físicas de Mantecadas Bajas en Grasa Almacenada en dos tipos de Material de Empaque durante su Vida de Anaquel
}

\author{
María del C. Beltrán-Orozco, Jorge H. Rendón Meza y Tzayhri Gallardo-Velázquez \\ Departamento de Graduados e Investigación en Alimentos, Escuela Nacional de Ciencias Biológicas, \\ Instituto Politécnico Nacional, Prolongación de Carpio y Pan de Ayala Col. Santo Tomás. \\ C. P. 11340. Delegación Miguel Hidalgo, México, D. F.-México (e-mail: cbeltran@encb.ipn.mx)
}

\begin{abstract}
Resumen
Se ha estudiado la vida de anaquel de mantecadas bajas en grasa y se ha comparado los resultados con los obtenidos para mantecadas con contenido completo de grasa (testigo). Las mantecadas bajas en grasa y las testigo se almacenaron bajo tres condiciones: sin material de empaque, en caja de poliestireno y en bolsa de polipropileno, durante un período de 168 horas, bajo las mismas condiciones de temperatura y de humedad relativa $\left(T_{a m b}=23^{\circ} \mathrm{C}, \mathrm{HR}=46 \%\right)$. Las variables evaluadas fueron: el peso, el contenido de humedad y el volumen y se obtuvo las curvas de cinética para cada unas de las variables de respuesta. El producto se mantuvo fresco durante $24 \mathrm{~h}$ sin material de empaque, $96 \mathrm{~h}$ en cajas de poliestireno y $168 \mathrm{~h}$ en bolsas de polipropileno, estos resultados fueron corroborados a través de las curvas de cinética obtenidas. La vida de anaquel de las mantecadas empacadas bajas en grasa resultó igual a las de las mantecadas testigo, bajo las mismas condiciones de almacenamiento.
\end{abstract}

\section{Kinetics of the Physical Characteristics of Low Fat Muffins Stored in two types of Packing Material during its Shelf Life}

\begin{abstract}
The storage life of low fat muffins have been studied and results compared to those obtained for whole muffins, with total fat content (standard). The low fat muffins and the standard ones were stored under three conditions: without packing materials, in polystyrene boxes, and in polypropylene bags at the same temperature and relative humidity $\left(T a m b=23^{\circ} \mathrm{C}, H R=46 \%\right)$. The following response variables were evaluated during the storage period: weight, water content and volume of the muffins and the kinetics of such variables was evaluated. Results showed that muffins maintained their freshness during 24 hours without packing material, $96 \mathrm{~h}$ in polystyrene boxes and $168 \mathrm{~h}$ in polypropylene bags. These results were also observed by the kinetic curves of the response variables. The storage live of the muffins with low and standard fat content were the same under the same storage conditions and packing material.
\end{abstract}

Keywords: low fat muffins, storage life, polystyrene, polypropylene, package material 


\section{INTRODUCCIÓN}

Las mantecadas son productos batidos, espumas, leudadas químicamente y cuya fuente de grasa es aceite, contienen una proporción de grasa se encuentra entre 15 y $25 \%$, respecto al peso total de todos los ingredientes.Tienen una corteza delgada, de color café-oro uniforme. La parte superior es simétrica, con un contorno similar al de la cabeza de una coliflor, con forma de hongo (champiñón). Los agujeros de la miga son redondos y de tamaño mediano, y las paredes de las celdas muy delgadas. Son ligeras y de miga suave (Charley, 1987). La vida de anaquel se define como el tiempo durante el cual un producto, generalmente envasado, permanecerá en buenas condiciones para ser vendido y consumido. Otro concepto que se utiliza en la comercialización de los alimentos, incluidos los productos de panificación, es la vida útil, que a su vez se define como el tiempo durante el cual un producto en su embalaje se puede comercializar en determinadas condiciones de almacenamiento, y es menor que la vida de anaquel (Bancomext aplicaciones glosario, en línea 2004, Glossary-Food Processing Technology, en línea, 2006).

\section{Envejecimiento del pan}

Durante el almacenamiento de los productos de panificación se produce un fenómeno denominado envejecimiento, el cual se manifiesta en un deterioro de la calidad del pan, con base al monitoreo de ese deterioro se determina cual es la vida útil y de anaquel de un producto. Los cambios que ocurren por el envejecimiento afectan: el peso, la humedad, el volumen, la textura, el contenido de almidón resistente y las características sensoriales. Durante el envejecimiento el almidón cambia lentamente, a temperaturas inferiores a $55^{\circ} \mathrm{C}$ se transforma de una estructura amorfa a una estructura cristalina; la consecuencia posterior, con menor humedad que al principio, es el rápido endurecimiento y encogimiento de los gránulos de almidón fuera de la estructura del gluten, estos cambios se conocen como retrogradación (Hoseney, 1994; Chinachoti, 2001). Se piensa que la formación de complejos de los polímeros de almidón, lípidos y proteínas de la harina inhibe la agregación de la amilasa y la amilopectina, reduciendo la velocidad de la retrogradación (Smith, J. et al. 2004), por lo que podría suponerse que la disminución de grasa en las mantecadas podría favorecer la retrogradación del pan, a menos que los imitadores de grasa formen complejos con los componentes del almidón que retarden la retrogradación del almidón de las mantecadas baja en grasa. Para retardar el envejecimiento del pan se pueden utilizar diversos materiales de empaque, usualmente fabricados a partir de películas plásticas ya que su costo es generalmente más económico que otros materiales de empaque; además, sus características físicas de resistencia mecánica, apariencia y barrera a los gases, permiten su uso en un gran número de productos (Rodríguez, 1997).

\section{Polipropileno y poliestireno, como materiales de empaque}

El polipropileno (PP) es un material de alta memoria, al doblarse éste tiende a recobrar la forma original. En película es un material altamente transparente, con gran resistencia a la punción y a la tensión, pero con baja resistencia al rasgado. El polipropileno bi-orientado (PP/BOPP) en forma de película es más económico y con altas propiedades de transparencia, barrera a la humedad, buena barrera contra gases y aromas, lo que permite una mayor vida de anaquel en los productos de panificación. No cambia sus características de protección en climas extremos, tiene estabilidad dimensional, baja electrostática, deslizamiento adecuado y rasgado uniforme, puede sellarse térmicamente y es inocuo (Vidales, 1995; Rodríguez, 1997; IMPI, 1997). Otro material de empaque es el poliestireno (PS) el cual se utiliza para fabricar artículos rígidos a partir de láminas del material por un proceso de termo formado, este material, presenta mínima barrera a gases o humedad, sin embargo, es ampliamente utilizado en la elaboración de estuches ya que su alta transparencia lo hace muy atractivo. EI PS es un material duro, aunque frágil, resistente a la tensión, de buena estabilidad dimensional y aislamiento eléctrico, además de ser inocuo (Rodríguez, 1997; IMPI, 1997). Dentro de los materiales de empaque más utilizados para la comercialización de los productos de panificación están son las bolsas de PP y las cajas rígidas de PS. Un pan empacado con una película plástica suele almacenarse dentro un período de hasta siete días, mientras se oferta al consumidor a este tiempo de almacenamiento se le llama vida de anaquel. Éste es un período en el que las características del pan aún son atractivas al consumidor 


\section{MATERIALES Y MÉTODOS}

\section{Diseño experimental}

Con la ayuda del programa estadístico Design Expert 5.0 (Stat-Ease Corporation. 1996) se elaboró un diseño experimental factorial $2^{2}$. Las variables de estudios fueron: la fórmula de la mantecada (baja grasa, grasa completa), y las condición de almacenamiento (sin material de empaque (SE), con material de empaque). Se hizo un diseño para cada uno de los materiales de empaque: caja de poliestireno (PS) y bolsa de polipropileno (PP). Metodología. Se elaboraron mantecadas con su contenido normal de grasa $(17.1 \%)$ las cuales fueron identificadas como mantecadas testigo $(T)$, la fuente de grasa para la preparación de dichas mantecadas fue aceite vegetal comestible. Las mantecadas bajas en grasa (BG), con un contenido de grasa del $4.3 \%$ (que corresponde a una reducción del $75 \%$ del contenido de grasa con respecto al testigo) se elaboraron utilizando dos diferentes sustitutos de grasa: maltodextrina y metilcelulosa (Beltrán-Orozco et al. 1998; BeltránOrozco et al. 2004).

\section{Condiciones de almacenamiento}

Las mantecadas se almacenaron en las siguientes condiciones: sin empaque (SE), en caja de poliestireno (PS) las cuales se cerraron de la misma manera que se acostumbran a comercializar y en bolsa de polipropileno (PP) selladas herméticamente con selladora térmica; bajo las mismas condiciones de temperatura y de humedad relativa $\left(T_{\mathrm{amb}}=23^{\circ} \mathrm{C}, \mathrm{HR}=46 \%\right)$. Durante un periodo de $168 \mathrm{~h}$ (7 días) se evaluaron las siguientes variables de respuesta: peso, volumen y humedad, de acuerdo con el diseño experimental elaborado. Los datos experimentales del estudio se ingresaron al diseño factorial para su optimación.

\section{Análisis fisicoquímico}

Se efectuaron las siguientes determinaciones por triplicado: i) Peso de la mantecada: Se cuantificó por medio de una balanza analítica. Al peso de la mantecada incluyendo el peso del papel del capacillo, se le sustrajo el peso del capacillo el cual era constante $(0.4 \mathrm{~g})$; ii) Volumen de la mantecada: Se determinó por un método modificado de desplazamiento de semillas (Beltrán-Orozco et al., 2004); Contenido de humedad: Se determinó utilizando un analizador de humedad (AACC, método 44-15A). El contenido de humedad, corresponde a la diferencia entre el peso inicial y final, de una muestra representativa de la mantecada, sometida a una temperatura de $130^{\circ} \mathrm{C}$; y iv) Velocidad de disminución de volumen, peso y humedad con respecto al tiempo de almacenamiento de las mantecadas.

Las cinéticas de pérdida de volumen, de peso y de humedad durante el almacenamiento de las mantecadas empacadas en diferentes tipos de empaque se obtuvieron aplicando una regresión polinomial de segundo orden a los datos experimentales de volumen, peso y humedad, en función del tiempo de almacenamiento, se obtuvieron valores de $\mathrm{R}^{2}$ en el intervalo de $0.95-0.99$. Estos polinomios fueron derivados con respecto al tiempo para obtener la función de la velocidad de pérdida de cada uno de los factores analizados respecto al tiempo de almacenamiento. Con estas funciones se obtuvieron las graficas de las cinéticas de perdida de las variables estudiadas.

\section{RESULTADOS Y DISCUSIÓN.}

Pérdida de peso y cinética de pérdida de peso de las mantecadas durante su almacenamiento.

En la Figura 1 se muestran los resultados de la pérdida de peso de las mantecadas con diferente nivel de grasa y almacenadas con diferentes materiales de empaque. La Figura 1 muestra que durante el período de almacenamiento el peso de las mantecadas disminuyó con respecto al tiempo. En el tratamiento sin empaque la pérdida de peso resultó importante después de las $24 \mathrm{~h}$ de almacenamiento. Los productos de panificación que contienen sustitutos de grasa, perdieron más peso que los productos sin sustitutos $(\alpha=0.05)$, debido al mayor contenido de humedad que 
proporcionan los sustitutos de grasa en forma de gel; esto concuerda con la mayor pérdida de peso observada en las mantecadas bajas en grasa respecto a las mantecadas testigo (ver Figura 1). Un comportamiento similar fue observado por Zúñiga (2000), en panes bizcocho con sustitutos de grasa almacenados sin empaque, donde se encontró una pérdida de peso importante a las $24 \mathrm{~h}$ de almacenamiento.

En el caso de las mantecadas almacenadas en bolsa de PP y en caja de PS se encontró una mayor pérdida de peso $(\alpha=0.05)$, en aquellas almacenadas en las cajas de poliestireno, a partir de las 96 $\mathrm{h}$ hasta las $168 \mathrm{~h}$ que duró el estudio (Figura 1). Las menores pérdidas de peso se registraron en el empaque con bolsa de PP (ver Figura 1) debido a la menor permeabilidad del polipropileno al vapor de agua $\left(3.1-7.75 \mathrm{~g} / \mathrm{m}^{2} \mathrm{~d}\right)$ respecto a la caja de PS $\left(77.5-155 \mathrm{~g} / \mathrm{m}^{2} \mathrm{~d}\right)$, lo cual permite que el producto empacado tenga una menor deshidratación en el mismo tiempo (IMPI, 1997) bajo las mismas condiciones de almacenamiento.

De Penna et al.(2003) estudiaron la vida útil de biscochuelos enriquecidos con fibra dietética y otros ingredientes almacenados en envases de polipropileno, durante 13 días (312 h) en condiciones ambientales $\left(25^{\circ} \mathrm{C}, 55-60 \% \mathrm{HR}\right)$, encontrado variaciones mínimas de peso, en dichas condiciones de almacenamiento, (la variación acumulada del peso máxima que encontraron fue de $0.04 \%$ del día 14 al 19 de almacenamiento), como se observó en este estudio para el mismo material de empaque (PP).

La pérdida de peso para las mantecadas baja en grasa (BG) fue de $1.5 \mathrm{~g}$ y para las mantecadas testigo $(\mathrm{T})$ fue de $1.1 \mathrm{~g}$. En el tratamiento con caja de PS, se observó una pérdida de peso con pendiente constante, muy semejante entre ambas fórmulas, de $5 \mathrm{~g}$ para las BG y de $4 \mathrm{~g}$ para las T. Muy semejante a la pérdida de $5 \mathrm{~g}$ observada por Zúñiga (2000) en panes tipo bizcocho con sustitutos de grasa, donde se utilizaron domos de poliestireno. Sin embargo para cada uno de los materiales de empaque estudiados no se encontró ningún efecto atribuible a la formula de la mantecada, lo que significa que la disminución en el contenido de grasa en la fórmula de las mantecadas no influye en la pérdida de peso durante el almacenamiento en dichos materiales de empaque. Una mantecada almacenada sin empaque tiene una vida de anaquel de $24 \mathrm{~h}$, mientras que dentro de la caja de PS se mantiene el producto con buenas características hasta $96 \mathrm{~h}$, y dentro de las bolsas de PP se mantiene fresco hasta las $168 \mathrm{~h}$ de almacenamiento.

La cinética de pérdida de peso que presentaron las mantecadas se muestra en la Figura 2, se puede apreciar que las mantecadas testigo (T-SE) y baja en grasa (BG-SE) almacenadas sin empaque, presentaron la mayor velocidad de pérdida de peso que con respecto a aquellas almacenadas con cualquier tipo de empaque durante todo el tiempo de almacenamiento. La velocidad de pérdida de peso para las mantecadas baja en grasa sin empaque (BG-SE), según la ecuación de la derivada del polinomio de $2^{\circ}$ orden se vuelve cero a un tiempo de almacenamiento de 150 horas, como se muestra en la cinética en la Figura 2, lo cual indicaría que a partir de este tiempo el peso de las mantecadas sin empaque se mantendría constante. El tiempo de almacenamiento donde la velocidad de pérdida de peso alcanza un valor de cero para las mantecadas testigo sin empaque, fue de 140 horas según su ecuación. La diferencia entre las BG y las T sin empaque se debe a la mayor pendiente (aceleración) de la cinética de pérdida de peso presentada por las BG y al menor peso en el equilibrio que alcanzan éstas respecto a las que las T, como se puede apreciar en la Figura 1. Con respecto a las mantecadas bajas en grasa empacadas con polipropileno (BG-PP) y poliestireno (BG-PS), se puede apreciar en la Figura 2, que sus velocidades de pérdida de peso son mayores que las velocidades presentadas por sus respectivos testigos a cualquier tiempo de almacenamiento.

Los cambios en el peso de los productos de panificación durante el almacenamiento son consecuencia de la redistribución del agua dentro del producto, así como las pérdida de agua por la evaporación al ambiente, esto ocurre a diferentes velocidades dependiendo de las condiciones de almacenamiento del producto (Primo-Martín, et al., 2006). Las velocidades de pérdida de peso para estos dos tipos de empaque tanto para las mantecadas bajas en grasa como para sus respectivos testigos, se mantienen prácticamente constantes en todo el periodo de almacenamiento, siendo 
ligeramente mayor la velocidad de pérdida de peso de las mantecadas empacadas con poliestireno. Esto indica nuevamente que el empaque de poliestireno favorece más la pérdida de peso de las mantecadas que el empaque de polipropileno. Las velocidades de pérdida de peso entre las mantecadas bajas en grasa y las correspondientes a sus testigos empacadas con polipropileno (BGPP y T-PP) son muy similares entre sí, como se aprecia en la Figura 2, lo cual indica que para el caso de este tipo de empaque, la disminución en grasa, no influye en la pérdida de peso de las mantecadas durante todo el periodo de almacenamiento en las condiciones de temperatura y humedad relativa estudiadas.

\section{Cinética de la pérdida de humedad de la mantecada, durante el almacenamiento.}

Uno de los factores clave que se relaciona con el envejecimiento del pan es el cambio en el contenido de humedad. El contenido de humedad inicial promedio de las mantecadas bajas en grasa fue de $42.12 \mathrm{~g} / 100 \mathrm{~g} \pm 1.52$; y el contenido de humedad inicial promedio de las mantecadas testigo fue de $28.24 \mathrm{~g} / 100 \mathrm{~g} \pm 2.20$. Las mantecadas bajas en grasa presentaron un mayor contenido de humedad, porque los sustitutos de grasa utilizados en forma de gel proporcionaron un mayor contenido de agua en las mismas. Resultados similares, con respecto al incremento de absorción de agua, por el uso de reemplazadores de grasa en forma de gel, fueron encontrados por O'Brien et al. (2003), al adicionar un gel de inulina como reemplazador de grasa para la elaboración de pan blanco, (a $2.5 \%$ y $5 \%$ ), las masas adicionadas del gel presentaron una mayor capacidad de absorción de agua que las masas control. $(\mathrm{P}<0.05)$. En la Figura 3 se muestran los datos del contenido de humedad promedio de las mantecadas, para cada uno de los tratamientos. Los datos de la Figura 3 muestran que durante el período de almacenamiento, el contenido de humedad de las mantecadas disminuyó con respecto al tiempo.

El contenido de humedad de ambas fórmulas disminuyó de manera semejante hasta las $96 \mathrm{~h}$ de almacenamiento $(\alpha=0.05)$, esto puede deberse a la habilidad de la metilcelulosa, uno de los imitadores de grasa usados en la fórmula, de actuar como un emulsionante (Bárcenas, 206). El estudio de Bárcenas (2006) mostró que los hidrocoloides formados por la hydroximetuilcelulosa (HMC) que utilizaron se integraron en la estructura de la miga, e interactuaron con sus constituyentes, ejerciendo un efecto protector. Los trabajos de Armero y Collar (1998), Crumb firming kinetics of wheat breads with anti-staling additives, y de Collar, Armero y Martínez (1998), Lipid binding of formula bread dough. Realationships with dough and bread technological performance, citados por Bárcenas (2006), sugieren la formación de redes físicas o enlaces reales entre la HMC y las proteínas del gluten o entre la HMC y los gránulos de almidón. Después de las $96 \mathrm{~h}$ de almacenamiento, la pérdida de humedad fue más notoria en el tratamiento sin empaque, las mantecadas bajas en grasa continuaron perdiendo humedad, mientras que las testigo presentaron un contenido de humedad prácticamente constante (ver Figura 3).

En el tratamiento con caja de poliestireno se observó una pérdida de humedad muy semejante entre ambas fórmulas $(\alpha=0.05)$. Esta pérdida resultó de $10 \mathrm{~g} / 100 \mathrm{~g}$ para las mantecadas bajas en grasa y de $11 \mathrm{~g} / 100 \mathrm{~g}$ para las mantecadas testigo. Muy semejante a la pérdida de $12 \mathrm{~g} / 100 \mathrm{~g}$, observada por Zúñiga (2000) en panes tipo bizcocho bajos en grasa, donde se utilizaron domos de poliestireno.

La menor pérdida de humedad se registró en el empaque con bolsa de polipropileno (ver Figura 3). Para las mantecadas bajas en grasa fue de $8 \mathrm{~g} / 100 \mathrm{~g}$, y para las mantecadas testigo fue de $5 \mathrm{~g} / 100 \mathrm{~g}$. Esto se debió a la menor permeabilidad del polipropileno al vapor de agua $(3.1-7.75 \mathrm{~g} / \mathrm{m} 2 \mathrm{~d})$, respecto a la caja de poliestireno $(77.5-155 \mathrm{~g} / \mathrm{m} 2 \mathrm{~d})$, ya que los materiales con barrera selectiva de humedad y de gases como el polipropileno, protegen de la pérdida de humedad a los productos (Smith, 2004). En el caso de la pérdida de la humedad, en cada tratamiento con empaque (PS y PP) se encontró una pérdida de humedad semejante entre ambas fórmulas (baja en grasa y testigo), no encontrándose diferencia significativa $(\alpha=0.05)$ entre ellas (Figura 3); lo que puede deberse a que los imitadores de grasa utilizados forman complejos con el producto que evitan la retrogradación prematura del almidón impidiendo la migración de la humedad de la miga hacia la costra (Miyazaki et al., 2004, Smith, et al. 2004). El envase en caja de PS, mantuvo la frescura del producto hasta $96 \mathrm{~h}$, mientras que la bolsa de PP hasta $168 \mathrm{~h}$ de almacenamiento. 


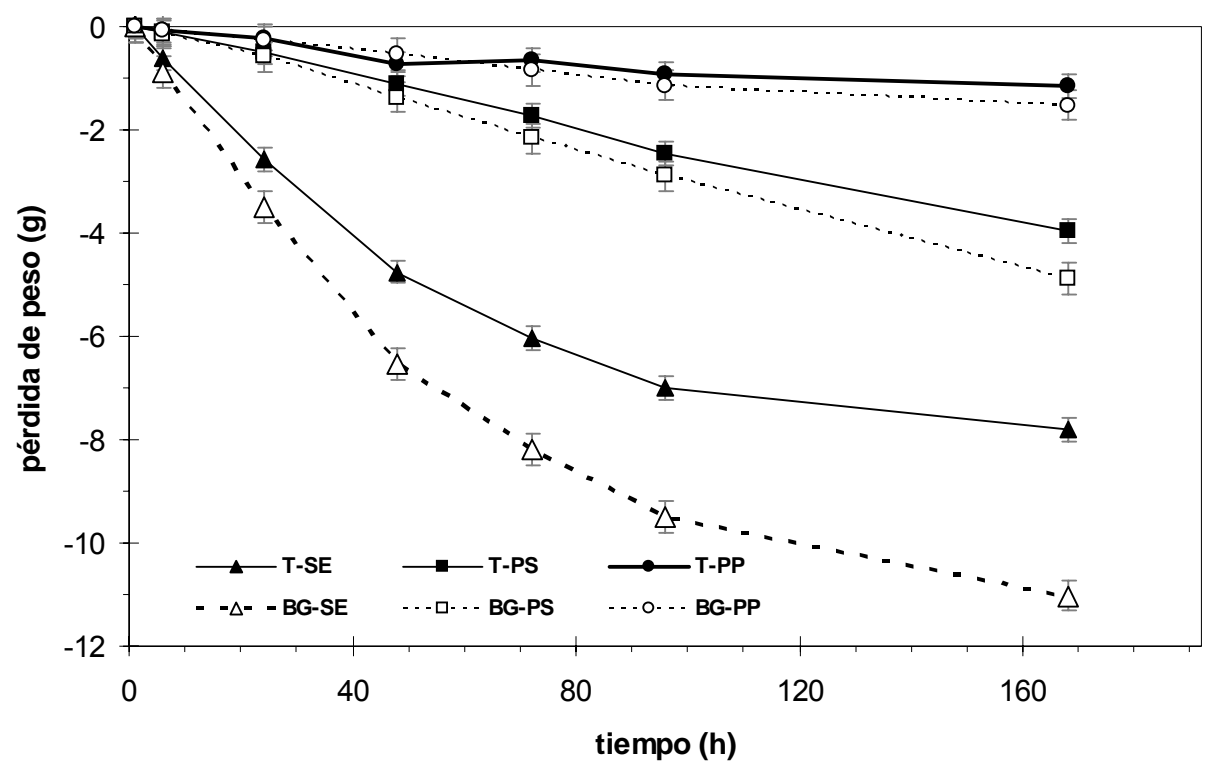

Fig. 1. Pérdida de peso de las mantecadas durante el almacenamiento

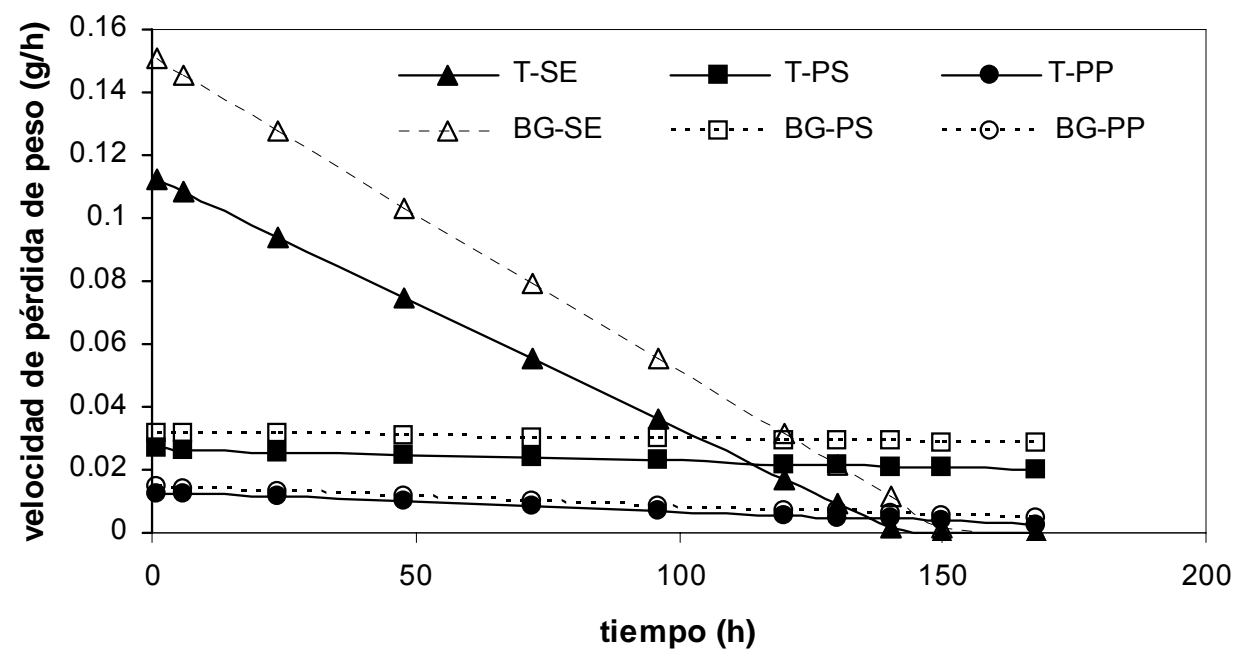

Fig. 2: Cinética de pérdida de peso de las mantecadas bajas en grasa y testigos sin empacar y empacadas con diferentes materiales

Jiang et al. (2005) en su estudio del efecto de la enzima xylanasa hiperthermofilica sobre la calidad del pan y el envejecimiento durante su almacenamiento; elaboraron panes adicionados de diferentes concentraciones de la enzima, los empacaron en bolsas de polipropileno y los almacenaron a temperatura ambiente, durante 6 días $(144 \mathrm{~h})$, encontraron que el incremento de la firmeza de la miga es uno de los cambios más evidentes durante el almacenamiento. Este incremento está asociado con la pérdida de peso y de humedad de los productos de panificación, que aún empacados en bolsas de polipropileno, sufren pérdidas de peso y de humedad, los cuales se hacen más evidentes a partir del cuarto día de almacenamiento (96 h), como es el caso de este estudio Fig. 1 y 3. La Figura 4 presenta la cinética de pérdida de humedad en función del tiempo de almacenamiento para las mantecadas bajas en grasa y sus respectivos testigos empacados con diferentes tipos de empaque.

Al igual que en caso de la Figura 2 (cinética de pérdida de peso), las mantecadas bajas en grasa y sus testigos sin empaque, son los productos que presentan las mayores velocidades de pérdida de humedad con respecto aquellas mantecadas que fueron empacadas con alguno de los materiales de empaque. Las mantecadas sin empacar bajas en grasa (BG-SE), presentaron velocidades mayores de pérdida de humedad que sus correspondientes mantecadas testigo (T-SE) durante todo el tiempo de almacenamiento. 
Sin embargo, la cinética de las mantecadas testigo (T-SE) presenta una pendiente (aceleración) mucho mayor que aquellas bajas en grasa (BG-SE). Por lo tanto, las mantecadas testigo (T-SE) presentaron una velocidad muy cercana a cero en un tiempo de aproximadamente $168 \mathrm{~h}$, alcanzando así el en grasa (BG-SE) lo lograrían en aproximadamente en 315 h según su ecuación, debido a que el equilibrio para estas muestras corresponde a contenidos de humedad mucho más bajos que para las mantecadas testigo con mayor contenido de grasa (Figura 3).

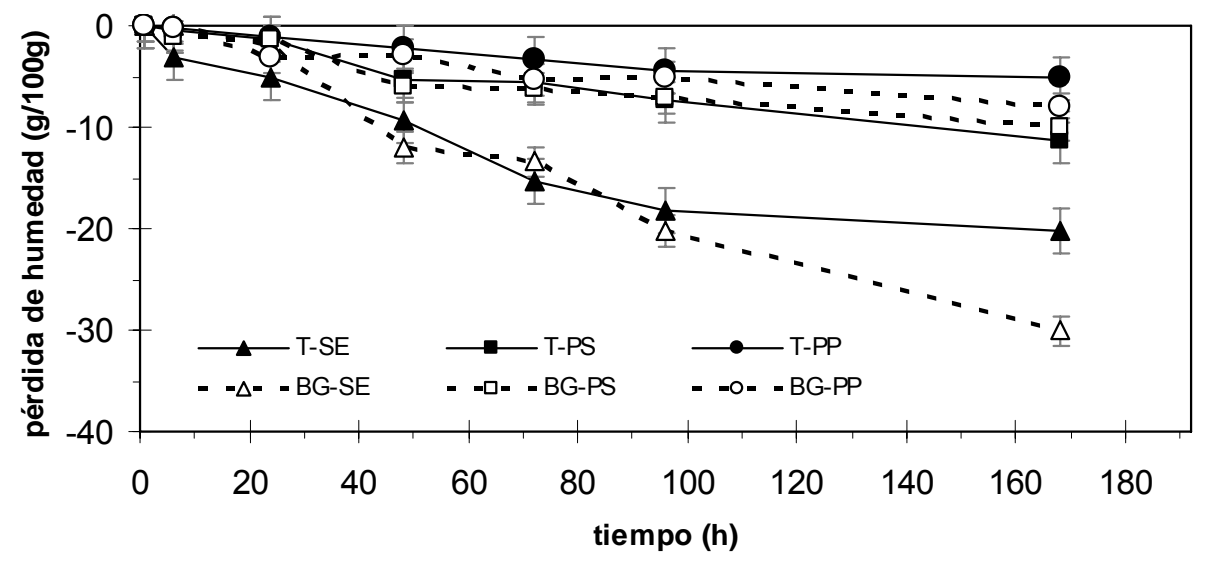

Fig. 3. Pérdida de humedad de las mantecadas durante el almacenamiento

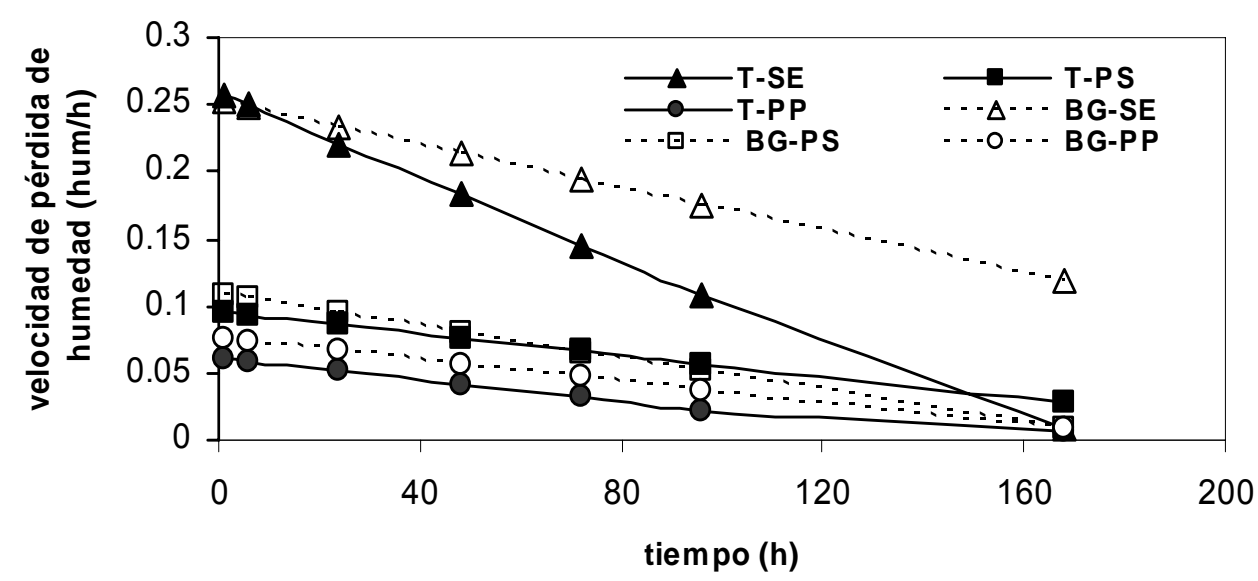

Fig. 4: Cinética de pérdida de humedad de las mantecadas bajas en grasa y testigos sin empacar y empacadas con diferentes materiales.

De las mantecadas empacadas con alguno de los materiales de empaque, las que fueron empacadas con polipropileno (BG-PP) y sus respectivos testigos (T-PP), fueron las que presentaron las menores velocidades de pérdida de humedad durante todo el tiempo de almacenamiento, siendo estas velocidades y sus respectivas aceleraciones muy similares entre sí. Las mantecadas empacadas con cajas de poliestireno y sus testigos presentaron mayores velocidades que aquellas empacadas con polipropileno durante todo el almacenamiento de las mantecadas, mostrando nuevamente que el polipropileno es el material de empaque más adecuado para las mantecadas bajas en grasa y testigo, no habiendo gran diferencia entre ellas.

Pérdida y cinética de la pérdida de volumen de la mantecada durante el almacenamiento.

El volumen inicial promedio de las mantecadas bajas en grasa fue de $130 \mathrm{~cm}^{3} \pm 2.55$; y el volumen inicial promedio de las mantecadas testigo fue de $135 \mathrm{~cm}^{3} \pm 2.52$. Las mantecădas bajas en grasa presentaron un menor volumen inicial que las mantecadas testigo, esto podría deberse a que los sustitutos de grasa formaron una estructura más firme durante el proceso de horneo. Esta estructura pierde humedad con más facilidad, por lo que ésta tiende a contraerse, lo que concuerda con la mayor disminución de volumen observada en la mantecada baja en grasa respecto a la mantecada 
testigo (ver Figuras 5 y 6 ). En todos los tratamientos (SE, PS, PP) la disminución más importante del volumen fue a las $24 \mathrm{~h}$ de almacenamiento, perdiéndose entre 10 y $15 \mathrm{~cm}^{3}$, como se puede apreciar en la Figura 5. En los casos con material de empaque, la pérdida de volumen no fue significativa $(\alpha=$ 0.05 ) entre fórmulas, solo fue significativa en el caso de las mantecadas sin material de empaque, a partir de las $96 \mathrm{~h}$ de almacenamiento. En la Figura 5, se puede notar que la disminución de volumen de las mantecadas testigo fue semejante entre los tres tratamientos (SE, PS, PP). Después de las 24 horas de almacenamiento el volumen no tuvo una pérdida significativa $(\alpha=0.05)$; el volumen permaneció prácticamente constante durante el resto del período de almacenamiento (la sensibilidad de método para medir el volumen es de $5 \mathrm{~cm}^{3}$ ).

La mayor pérdida de volumen en las mantecadas bajas en grasa, se encontró en las almacenadas sin material de empaque, ya que al perder humedad, la estructura formada por los sustitutos de grasa se contrae. En aquellas almacenadas en material de empaque, la pérdida de volumen fue significativamente menor. Hasta las $96 \mathrm{~h}$ del estudio, no se observó diferencia significativa $(\alpha=0.05)$ entre la pérdida de volumen de las mantecadas bajas en grasa almacenadas en poliestireno y en polipropileno, solo hasta las $168 \mathrm{~h}$ se observa una mayor pérdida de volumen en las almacenadas en caja de poliestireno (Figura 6).

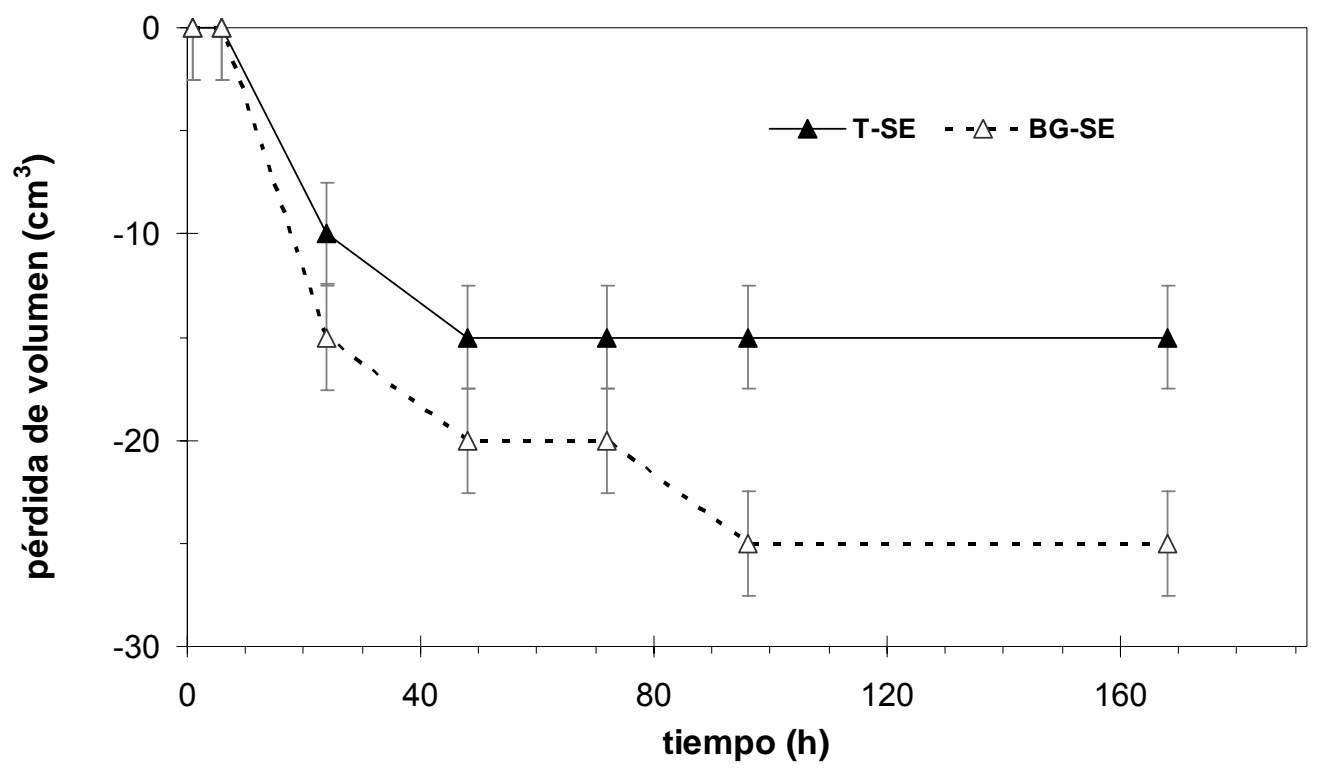

Fig. 5: Pérdida de volumen de las mantecadas testigo y bajas en grasa sin empaque, durante el almacenamiento

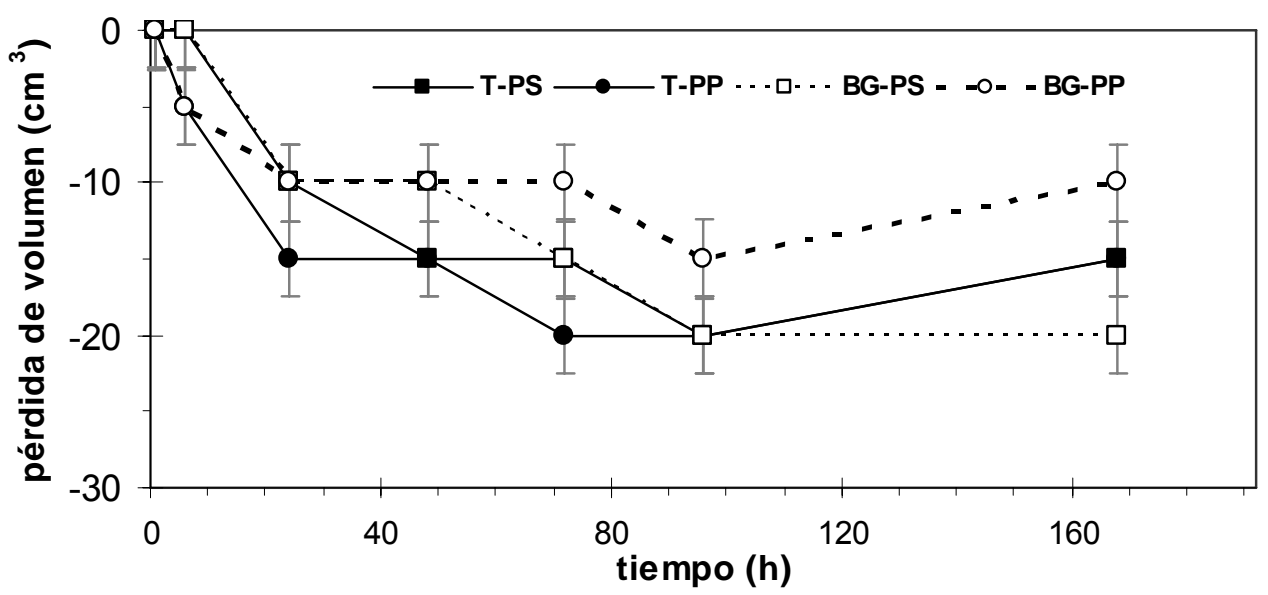

Fig. 6. Pérdida de volumen de las mantecadas testigo y bajas en grasa con empaque, durante el almacenamiento 
Las mantecadas bajas en grasa almacenadas en material de empaque perdieron el mismo volumen que las testigo durante el período de estudio, en las mismas condiciones de almacenamiento $\left(T_{\text {amb }}=\right.$ $23^{\circ} \mathrm{C} ; \mathrm{HR}=46 \%$.). Esta variable no tuvo un alto impacto, ni en la apariencia del producto, ni en las características sensoriales del mismo. El material de empaque conserva la humedad del producto por más tiempo, por lo que la estructura formada por los sustitutos de grasa no se contrae de manera significativa $(\alpha=0.05)$, y se mantiene estable. Con respecto a las velocidades de disminución de volumen de las mantecadas, en la Figura 7 se presenta la cinética de pérdida de volumen de las mantecadas bajas en grasa y testigos empacadas con diferentes tipos de empaque, así como aquellas sin empacar.

Como en los dos casos anteriores (pérdida de peso y humedad), las mantecadas sin empaque bajas en grasa (BG-SE), presentaron las mayores velocidades de disminución de volumen en comparación a aquellas presentadas por las mantecadas empacadas (BG-PP y BG-PS) durante el almacenamiento (ver Figura 6). La Figura 6 muestra que las mantecadas empacadas con polipropileno (BG-PP) fueron las que presentaron las menores velocidades de disminución de volumen en todo el tiempo de almacenamiento. Esta figura también muestra que las mantecadas bajas en grasa con o sin empaque (BG-SE, BG-PS y BG-PP) alcanzaron una velocidad cero de disminución de volumen a las $48 \mathrm{~h}$ de almacenamiento, por lo que el volumen se mantiene constante a partir de este tiempo, mientras que las mantecadas testigo sin empacar (T-SE) y empacadas en cajas de poliestireno (T-PS) alcanzaron el tiempo de velocidad cero a las 72 horas de almacenamiento, por lo que sufrieron un mayor encogimiento.

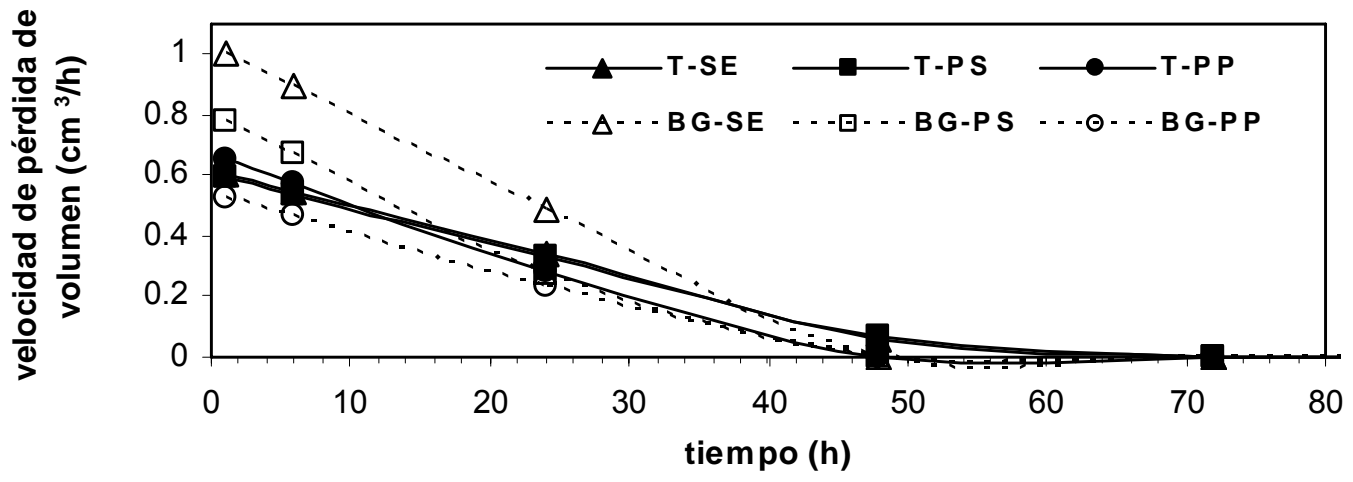

Fig. 7: Cinética de pérdida de volumen de las mantecadas bajas en grasa y testigos sin empacar y empacadas con diferentes materiales.

\section{CONCLUSIONES}

En los productos almacenados sin material de empaque las características de peso, volumen y humedad de la hogaza, tuvieron una mayor disminución en las mantecadas bajas en grasa. La pérdida de volumen más importante fue a las $24 \mathrm{~h}$ de almacenamiento. La velocidad (dx/dt) de pérdida de peso, de volumen y de humedad de las mantecadas, presentaron la misma secuencia de mayor a menor velocidad: BG-SE>>BG-PS>BG-PP. El empacado de las mantecadas bajas en grasa en bolsas de polipropileno fue la que mantuvo as características iniciales de estas mantecadas durante un mayor tiempo. Las velocidades de pérdida de peso y humedad, de las mantecadas bajas en grasa con empaque (BG-PP y BG-PS), fueron muy similares a sus correspondientes testigos con contenido normal de grasa (T-PP y T-PS), por lo que no hay diferencia apreciable en estas variables entre las mantecadas bajas en grasa y testigos. Las cinéticas de pérdida de volumen de las mantecadas bajas en grasa (BG-SE, BG-PS) y los testigos con cualquiera de los empaques alcanzaron el punto de velocidad de disminución de volumen cero hasta las 72 horas de almacenamiento, por lo que sufrieron un mayor encogimiento.

Las variables respuesta más afectadas por el envejecimiento fueron la humedad y el peso de las mantecadas. Para cada uno de los materiales de empaque estudiados no se encontró ningún efecto atribuible a la formula de la mantecada. La vida de anaquel de las mantecadas de ambas fórmulas resultó igual, a las mantecadas testigo, cuando se almacenaron con empaque, bajo las mismas 
condiciones de almacenamiento. La vida de anaquel de las mantecadas, tanto las bajas en grasa como las testigo, fue de $24 \mathrm{~h}$ para aquellas almacenadas sin material de empaque, $96 \mathrm{~h}$ para las almacenadas en cajas de poliestireno y $168 \mathrm{~h}$ para las almacenadas en bolsas de polipropileno

\section{REFERENCIAS}

AACC. Approved Methods of the AACC; AACC: Method 08-01, Method 10-05, Method 30-10, Method 4415A, Method 54-30A (2003).

Bancomext aplicaciones glosario [en línea] 2004. http://www.bancomext.com. Acceso 10 de Octubre (2006).

Bárcenas, M. E. y Rosell, C. R. Different approaches for improving the quality and extending the shelf life of the partially baked bread: low temperatures and HPMC addition. . J. of Food Eng. 72, (1), 92-99 (2006).

Beltrán-Orozco, M. C.; Mares, J. C., Jiménez, E. y Hernández-Sánchez, H., Development of a low fat muffin. Institute of Food Technol. Annual Meeting. June 20-24, 1998, Atlanta, Georgia, USA (1998).

Beltrán-Orozco, M. C., Rendón, J. H. y Mares, J.C., Desarrollo de una fórmula para la elaboración de mantecadas bajas en grasa. Industria Alimentaria. 26 (6), 10-18 (2004).

Charley, H., Tecnología de Alimentos. Editorial Limusa-Noriega, México (1987).

Chinachoti, P. y Vodovotz, Y., Bread staling. CRC Press LLC. Boca Ratón, Florida-USA. (2001).

De Penna, E., Avendaño, P., Soto, D. y Bunger, A., Caracterización química y sensorial de biscochuelos enriquecidos con fibra dietética y micronutrientes para el anciano. Archivos Latinoamericanos de Nutrición. 53 (1), 74-83 (2003).

Glossary-Food processing Technology [en línea], 2006._http://www.foodprocessing-technology.com/ Acceso: 1 Octubre (2006).

Hoseney, R. C., Principles of cereal science and technology. $2^{\mathrm{a}}$ edición. American association of cereal chemists, Inc. St. Paul, Minnesota, USA (1994).

IMPI. Enciclopedia del plástico. Instituto Mexicano del Plástico Industrial, S. C., México (1997).

Jiang, Z., Li, X., Yang, Sh. y Tan, S., Improvement of the breadmaking quality of wheat flour by the hyperthermophilic xylanase $B$ from Thermotoga maritima. Food Research Int. 38, (1), 37-43 (2005).

Miyazaki, M., Maeda, T., y Morita, N., Effect of various dextrin substitutions for wheat flour on dough properties and bread qualities. Food Research Internacional, 37, (1), 59-65. 2004

O'Brien, C. M., Muller, A., Scannell, A. G. M. y Arendt, E. K., Evaluation of the effects of a fat replacers on the quality of wheat bread. Journal of Food Engineering 56, 265-267 (2003).

Primo-Martín, C., van de Pijpekamp, A., van Vliet, T., de Jongh, H., Plijter, J. y Hamer, R. The rol of the gluten network in crispness of read crust. Journal of Cereal Science. 43, (3), 342-352. (2006).

Rodríguez, J. A., Manual de ingeniería y diseño en envase y embalaje. $3^{a}$ edición. Packing: Ingeniería en envase y embalaje. México (1997).

Smith, J. P., Daifas, D. P., El-Khoury, W. y Koukoutsis, J., Critical Reviews in Food Science and Nutrition 44, 19-55. (2004)

Stat-Ease Corporation. Design Expert 5.0, Reference Manual. 2021 East Hennepin Avenue, Suite 191, Minneapolis-USA MN 55413 (1996).

Vidales, M. D., El mundo del envase: manual para el diseño y producción de envases y embalajes. Editorial Gustavo Gili, S.A. de C. V. México (1995).

Zúñiga, M. C., Estudio comparativo de cinéticas de envejecimiento en pan bizcocho "bollo" adicionado con imitador de grasa y fibra. Tesis de licenciatura carrera Ingeniero Químico. Benemérita Universidad Autónoma de Puebla. Puebla-México (2000). 